\title{
Positive Sequence Voltage Control, Full Negative Sequence Cancellation and Current Limitation for Static Compensators
}

\author{
Antonio Camacho, Miguel Castilla, Jaume Miret, Member, IEEE, Manel Velasco and Ramon Guzman.
}

\begin{abstract}
This paper presents a control scheme in the stationary reference frame for Static Synchronous Compensators that provides three features: 1) regulation of the positive sequence voltage to the nominal value, 2) full cancellation of the negative sequence voltage, and 3) peak limitation of the injected currents. These features can help improving the performance of these devices under perturbed grid conditions, including low, high and unbalanced voltages. Also, the peak current limiter guarantees both the safe operation of the converter, and a priority-based achievement of the voltage regulation targets, which is of interest during severe perturbations like voltage dips. Experimental results, implementation details and closed-loop analysis are presented to validate the regulation capabilities and to demonstrate the feasibility of the power quality improvement.
\end{abstract}

Index Terms-Reactive power control, grid fault, voltage dip, voltage sag, voltage support, positive and negative sequence control.

\section{INTRODUCTION}

$\mathbf{P}$ OWER quality is a major concern for distribution and transmission system operators. Environmental issues and economical constraints have inspired new methods for controlling the grid-tied elements that are present in the electrical system [1], [2]. Among these new methods, there exists a noticeable interest in extending the use of power electronics converters to improve the power quality of the grid and to facilitate the integration of renewable and cleaner distributed generation sources [3], [4].

Static Synchronous Compensators (STATCOMs) are power electronics equipments that provide fast reactive power. They can be located close to distributed generation power plants or in selected places where the grid need to be precisely supported. The main objectives of these devices are to increase the controllability of the system, to reduce losses, to modify the power factor in selected locations, to regulate the voltage and to support the grid during voltage dips [5]. Moreover, the massive integration of power electronics equipments into the grid, has resulted in a new generation of ancillary services for

This work was supported in part by the Ministry of Science, Innovation, and Universities of Spain and in part by the European Regional Development Fund under Project RTI2018-100732-B-C22. (Corresponding author, Antonio Camacho).

A. Camacho, M. Velasco and R. Guzman are with the Department of Automatic Control, Technical University of Catalonia, 08028 Barcelona, Spain (e-mail: antonio.camacho.santiago@upc.edu; manel.velasco@upc.edu; ramon.guzman@upc.edu).

M. Castilla and J. Miret are with the Department of Electronic Engineering, Technical University of Catalonia, 08800 Vilanova i la Geltrú, Spain (e-mail: miquel.castilla@upc.edu; jaume.miret@upc.edu) distributed STATCOMs. Harmonic compensation, voltage imbalance mitigation, inertia emulation or damping oscillations, are some of the new functionalities that power plants shall attend in future grid codes [6]-[8].

In this context, an advanced control for a STATCOM is presented in this work. The proposed controller is focused on three main control objectives: 1) to regulate the positive sequence voltage to the nominal value, 2) to completely cancel the negative sequence voltage, and 3) to implement a peak current limiter with priority assignment that ensures a safe operation of the STATCOM in any circumstance. The control proposed for the STATCOM is developed in the stationary reference frame, and uses the symmetric sequences tool to achieve these objectives. In ideal grid conditions, the threephase voltages should be balanced at the nominal value, which translates into a positive symmetric sequence equal to 1 per unit (p.u.), and a negative symmetric sequence equal to zero. These are the desired voltage objectives of the proposed control scheme.

The main challenge of the proposed control is the full cancellation of the negative sequence voltage, which is a common perturbation in electrical systems. The main cause of such a problem arises from the unbalanced loads across the distribution system. This perturbation causes power quality issues as for example pulsating torque in motor loads, which deteriorates its operation and life-cycle [9], [10], or higher power losses due to the overheating phenomenon [10], [11]. The full cancellation of the voltage imbalance with conventional control algorithms in the $\alpha \beta$ reference frame presents some problems. In particular, the numerator and denominator of the reference currents are built from a voltage that tends to vanish as the measured voltage tends to zero, thus causing the reference currents to become undetermined and loosing the synchronization in the injected currents. This problem will be highlighted in Section II-C, and the proposed solution to properly address this issue will be presented in Section III.

Full cancellation of negative sequence voltage has been briefly discussed in the literature [12]-[21]. Some of these works [12]-[15] cannot cancel the voltage imbalance completely, but until a certain limit due to the loss of synchronism previously mentioned. Other research works [16]-[18], present only simulation results, or provide few details regarding the implementation and the results obtained [19], [20]. Also, some interesting works are presented in [18], [20], [21] although these investigations are not based on sequence extractors, but on indirect power-based methods or in the machine model of 
TABLE I: Comparison with State-of-the-Art

\begin{tabular}{|c|c|c|c|c|c|c|c|}
\hline Ref. & $\overline{V^{+}}=1$ & $V^{-}=0$ & Current limit & Dips/Swells & $L$ knowledge & Complexity & Application \\
\hline [13] & $\checkmark$ & $x$ & $x$ & $x$ & $\checkmark$ & Medium & single STATCOM \\
\hline [14] & $\checkmark$ & $\checkmark$ & $x$ & 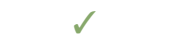 & $x$ & Medium & single STATCOM \\
\hline [15] & $x$ & $x$ & $x$ & $\checkmark$ & $x$ & Low & coordinated microgrid \\
\hline [16] & $\checkmark$ & $\checkmark$ & $x$ & $x$ & $x$ & Low & single inverter \\
\hline [17] & $x$ & $\checkmark$ & $\checkmark$ & $x$ & $x$ & Medium & single DFIG \\
\hline [18] & $\checkmark$ & $\checkmark$ & $\checkmark$ & $x$ & $x$ & Low & single STATCOM \\
\hline [19] & $\checkmark$ & $\checkmark$ & $\checkmark$ & $x$ & $x$ & Medium & single back-to-back \\
\hline [20] & $x$ & $\checkmark$ & $x$ & $x$ & $\checkmark$ & Medium & single DFIG \\
\hline [21] & $x$ & $x$ & $\checkmark$ & $x$ & $\checkmark$ & Low & single inverter \\
\hline [22] & $x$ & $\checkmark$ & $x$ & $x$ & $\checkmark$ & Medium & single inverter \\
\hline [23] & $x$ & $\checkmark$ & $x$ & $x$ & $\checkmark$ & High & coordinated microgrid \\
\hline Proposal & $\checkmark$ & $\checkmark$ & $\checkmark$ & $\checkmark$ & $x$ & Low & single STATCOM \\
\hline
\end{tabular}

double fed induction generators. Finally, [22], [23] are focused on cancelling the negative sequence voltage in microgrids, although the controller is not suited to ride through voltage dips or swells, contrary to the proposed work.

Table I collects the main characteristics of state-of-the-art works to clearly highlight the differences of the proposal with the existing research. Several topics are analyzed in this table. In particular it has been compared the capability to regulate positive sequence voltage, negative sequence voltage and peak current limitation, which are the three main control objectives of the proposal. Also, the capability to ride through voltage dips or swells, the grid impedance knowledge and dependence, the implementation complexity and the application where the control is applied have been considered for the comparison. The table shows that for the given control objectives proposed in the work, the proposal presents interesting features for STATCOM applications. As a drawback of the proposed work, the control scheme requires a certain knowledge and it is dependent on the grid impedance, which is a common drawback when supporting the voltage during sudden perturbations as grid faults.

The proposed control scheme developed along this work uses a simple algorithm to fully cancel the negative sequence voltage by using a typical symmetric sequence controller. In fact, one of the main features of the proposal compared with previous works is its simplicity, since the typical structure of independent positive and negative sequence controllers is used, the tuning of the controller is straightforward, and the performance is fairly good with a low complexity. This objective is accomplished by using a virtual voltage estimator that allows the full cancellation of the voltage imbalance. Also, the proposal is developed in the stationary reference frame, which constitutes a new development in this research area, as opposed to most of the state-of-the-art proposals which are based on synchronous reference frame controllers.

The next part of the work is related to the third control objective, the safe operation of the STATCOM in front of sudden grid perturbations as for example voltage dips or swells. Under these severe perturbations, the converter could trip by over-current. To avoid this drawback, a peak current limiter is developed. The main characteristic of the peak current limiter is to avoid injecting more current in any phase than the maximum rated current of the power converter. Several peak currents limiters have been proposed in the literature [24][29]. Some of them are used for the particular objective of cancelling dc-link oscillations [27]. The limiters in [28], [29] are quite complex and generalized for maximum admissible total active or reactive powers, requiring extensive computational resources. However, the proposed algorithm is implemented in two steps yielding to a priority-based development, which constitutes a new contribution in this area. The first priority of the limiter is to regulate the positive sequence voltage, and the second priority is to cancel the voltage imbalance. This methodology is aligned with typical grid operator needs. As a result, a low complexity computation of the maximum positive and negative reactive currents is obtained, ensuring that the injected phase currents will not surpass a given threshold.

To summarize the main control objectives developed along this work, the first one is the positive sequence voltage regulation, the second one is the full cancellation of the negative sequence voltage, and the the third one is the peak current limiter. The second control objective constitutes the main contribution of the work, since it provides enhanced power quality characteristics to the STATCOM. The third objective is the second contribution of the work and helps to safely operate STATCOMs during any grid voltage perturbation. Finally, it should be highlighted that these three control objectives have not been previously combined in one single implementation and they provide advanced features for improving the power quality of the grid. Theoretical analysis and practical implementation issues are widely discussed, including the closed-loop analysis. Also, experimental results are provided to demonstrate the power quality improvement in a low-power scaled-down laboratory prototype.

The rest of the paper is organized as follows, Section II presents the plant and the control scheme of the STATCOM. Section III develops the proposed controller. Section IV presents the closed-loop analysis. Section V shows the experimental results. Finally, Section VI concludes the paper.

\section{Problem Statement}

This section presents the basics for the plant and the control of the STATCOM. It introduces the main control objectives and the issues related to conventional controllers when cancelling the negative sequence voltage. 


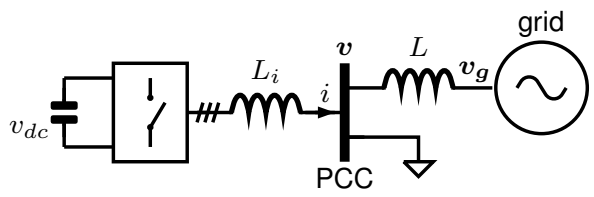

Fig. 1: Plant model of the STATCOM.

\section{A. Plant}

Fig. 1 shows the plant scheme of the STATCOM. which is made of a full bridge voltage-source converter with an inductor $L_{i}$ to reduce switching harmonics. The grid is modelled as an unbalanced voltage source connected to the STATCOM at the point of common coupling (PCC). In between the power converter and the grid, an equivalent inductance $L$ represents the grid impedance. Finally, a local load is attached at the PCC.

\section{B. Control Objectives}

The control objectives developed for the STATCOM can be formulated as

$$
\begin{gathered}
V_{\text {ref }}^{+}=1 \text { p.u. } \\
V_{\text {ref }}^{-}=0 \text { p.u. } \\
\max \left\{I_{a}, I_{b}, I_{c}\right\} \leq I_{\max .}
\end{gathered}
$$

The first control objective in (1) is in charge of regulating the positive sequence voltage to 1 p.u. This means that whenever the positive sequence voltage surpasses the nominal value, lagging reactive power will be compensated by the STATCOM. Similarly, for low positive sequence voltage, leading reactive power will be injected to increase the voltage. It should be noticed that other voltage objectives can also be scheduled during daily peak or valley scenarios to improve the system performance, although 1 p.u. is selected for simplicity.

The second objective in (2) tries to remove the negative sequence voltage at the terminals of the STATCOM. Thus, cancelling the voltage imbalance and improving the power quality of the system.

The third objective in (3) implies that the maximum phase current provided by the STATCOM must be safely limited to the maximum rated current of the converter $I_{\max }$. Then, it is guaranteed a safe operation that avoids the over-current tripping. This peak current limiter with priority assignation is devoted to extend the applicability of the control proposal not only to conventional operation modes, but also to balanced and unbalanced voltage swells and dips of any severity, independently of the stiffness/weakness of the grid.

\section{Control Issues in Conventional Reference Generators}

As stated above, the control proposal is developed in the stationary reference frame. Clarke's transformation [30] is used to translate the phase voltages and currents into the corresponding $\alpha \beta$-components.

Conventionally, a positive and negative sequence reference generator has been employed to inject positive and negative sequence reactive currents in the STATCOM. For doing this, a sequence extractor is required. For a detailed explanation of the sequence extractor, a literature review and the implementation details, see [31]-[33], and the references therein. Along this work, second order generalized integrators (SOGI) will be used as the sequence extractor [33]. Reference generators based on symmetric sequences consist of four terms, two related to the positive sequence component, and two for the negative one. Each one of these parts is also divided in two more pieces, one related to the amplitude of the current to be injected, and the other part related to the sinusoidal shape. A conventional current reference generator [25] used for this purpose is given in the following

$$
\begin{aligned}
& i_{\alpha}^{*}=I_{q}^{+} \frac{v_{\beta}^{+}}{V^{+}}+I_{q}^{-} \frac{v_{\beta}^{-}}{V^{-}} \\
& i_{\beta}^{*}=-I_{q}^{+} \frac{v_{\alpha}^{+}}{V^{+}}-I_{q}^{-} \frac{v_{\alpha}^{-}}{V^{-}}
\end{aligned}
$$

where $i_{\alpha}^{*}$ and $i_{\beta}^{*}$ are the reference currents in the $\alpha \beta$-channels, $I_{q}^{+}$and $I_{q}^{-}$are the amplitude of the positive and negative reactive currents respectively and need to be computed based on the desired control objective, $v_{\alpha}^{+}, v_{\beta}^{+}, v_{\alpha}^{-}$and $v_{\beta}^{-}$are the instantaneous positive and negative voltages at the PCC after being processed by a sequence extractor, and $V^{+}$and $V^{-}$are the amplitude of the positive and negative sequence voltages computed as

$$
\begin{aligned}
V^{+} & =\sqrt{\left(v_{\alpha}^{+}\right)^{2}+\left(v_{\beta}^{+}\right)^{2}} \\
V^{-} & =\sqrt{\left(v_{\alpha}^{-}\right)^{2}+\left(v_{\beta}^{-}\right)^{2}} .
\end{aligned}
$$

However, there is a problem with the above presented reference generator in (4) and (5). This reference generator is unable to fully cancel the negative sequence voltage because when the instantaneous negative sequence voltages $v_{\alpha}^{-}$and $v_{\beta}^{-}$tend to dissapear, then their amplitude $V^{-}$also tends to zero, and the reference generator becomes undetermined. In such a case, both the amplitude and the shape of the negative sequence terms in (4) and (5) tend to zero, which causes the reference currents to loss the synchronization with the negative sequence voltages.

A simulation under these conditions, based on the reference generator in (4) and (5), is presented in Fig. 2(a). In this plot, the injected currents and the negative sequence voltage amplitude at the grid $V_{g}^{-}$and the PCC side $V^{-}$are shown. It can be appreciated how the controller works as expected (i.e. the negative sequence voltage is being reduced) until reaching a very low voltage value. After this point, the reference current generator fails and the injected currents contain harmonics and cannot cancel the negative sequence voltage. This is a common problem of some controllers that use a measured magnitude that vanishes to build a reference signal. As a result, the reference should be zero. But in the case under discussion, the injected negative sequence reactive current cannot be null when trying to cancel the negative sequence voltage. Therefore, the conventional reference generator must be improved to fix this issue.

A new simulation with the control proposal developed in this work, fixing the issues shown in the previous simulation, is presented in Fig. 2(b). Note that the amplitude of the negative 

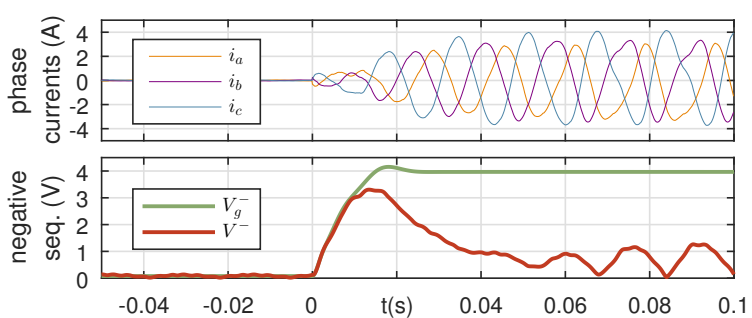

(a)
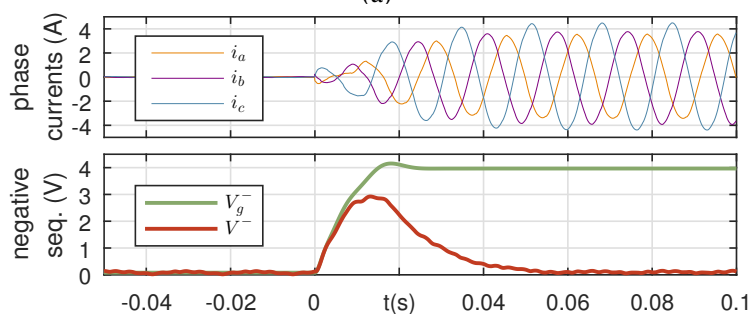

(b)

Fig. 2: Simulation results for (a) the conventional, and (b) the proposed implementation. Top: injected phase currents. Bottom: Negative sequence voltage amplitudes at the grid side $\left(V_{g}^{-}\right)$and at the PCC $\left(V^{-}\right)$.

sequence at the PCC tends to zero, and the injected currents lack of harmonics. The step-by-step realization of the proposal is given in next Section.

\section{Control Proposal}

The proposed control scheme to fix the above mentioned issue is based on the generation of a virtual voltage that does not vanish when the negative sequence voltage is cancelled, which is the basis for the generation of a reference voltage that can effectively cancel the negative sequence voltage as will be shown in the following.

\section{A. Virtual Voltage}

The virtual voltage needed for the proposed reference generator is built based on the dynamic model of the plant presented in Fig. 1.

$$
\begin{aligned}
& \widehat{v}_{\alpha}=v_{\alpha}-\widehat{L} \frac{d i_{\alpha}}{d t} \\
& \widehat{v}_{\beta}=v_{\beta}-\widehat{L} \frac{d i_{\beta}}{d t}
\end{aligned}
$$

where $\widehat{v}_{\alpha}$ and $\widehat{v}_{\beta}$ are the virtual voltages in the stationary reference frame, $v_{\alpha}, v_{\beta}, i_{\alpha}$ and $i_{\beta}$ are the Clarke transformation of the measured voltages and currents respectively, and $\widehat{L}$ is a virtual inductance, needed for the control scheme. Note that the virtual voltage exists only inside the controller, and emulates the voltage seen at any given distant point far from the converter. The purpose of the virtual voltage is to avoid loosing the synchronization both in the numerator and the denominator of (4) and (5) when approaching zero, resulting in the adverse effect shown in Fig. 2(a). The virtual voltages $\widehat{v}_{\alpha}$ and $\widehat{v}_{\beta}$ obtained from (8) and (9) have some interesting features that make the control proposal capable to fully cancel the negative sequence voltage. The main characteristic is that the virtual voltages are in phase with the original measurements, due to the fact that the STATCOM only injects reactive current. However, the amplitudes of virtual and measured values are slightly different, depending on the virtual inductance and the injected currents. Moreover, even when the measured voltages are zero, the virtual voltages used in the reference generator keep the appropriate phase to inject the desired reactive power, since they are reproducing a distant point where the amplitude is non-zero. Therefore, these characteristics avoid the loss of synchronization in the reference currents and allows the STATCOM to fully cancel the negative sequence voltage.

In fact, the virtual voltage estimator provides enhanced characteristics to the closed-loop system as will be shown in Section IV. Also, note that the time derivative used in the virtual voltage computation does not have adverse effects within the control loop (this term normally introduces highfrequency noise in the control algorithm) due to the fact that it is post-processed by a sequence extractor, as will be shown below.

\section{B. Sequence Extractor}

It should be noticed that the virtual voltage must be filtered to remove noise and harmonics. The symmetric sequence extractor performs this filtering and also computes the positive and negative instantaneous virtual voltages in the stationary reference frame $\widehat{v}_{\alpha}^{+}, \widehat{v}_{\beta}^{+}, \widehat{v}_{\alpha}^{-}, \widehat{v}_{\beta}^{-}$.

Similarly as in (6) and (7), the amplitudes of the positive and negative sequence virtual voltages are obtained as

$$
\begin{aligned}
& \widehat{V}^{+}=\sqrt{\left(\widehat{v}_{\alpha}^{+}\right)^{2}+\left(\widehat{v}_{\beta}^{+}\right)^{2}} \\
& \widehat{V}^{-}=\sqrt{\left(\widehat{v}_{\alpha}^{-}\right)^{2}+\left(\widehat{v}_{\beta}^{-}\right)^{2}} .
\end{aligned}
$$

In addition, the angle between the positive and the negative sequence virtual voltages $\widehat{\varphi}$ can be obtained as

$$
\begin{aligned}
& \cos \widehat{\varphi}=\frac{\widehat{v}_{\alpha}^{+} \widehat{v}_{\alpha}^{-}-\widehat{v}_{\beta}^{+} \widehat{v}_{\beta}^{-}}{\widehat{V}^{+} \widehat{V}^{-}} \\
& \sin \widehat{\varphi}=\frac{\widehat{v}_{\alpha}^{+} \widehat{v}_{\beta}^{-}+\widehat{v}_{\alpha}^{-} \widehat{v}_{\beta}^{+}}{\widehat{V}^{+} \widehat{V}^{-}} \\
& \widehat{\varphi}=\operatorname{atan} 2(\sin \widehat{\varphi}, \cos \widehat{\varphi})
\end{aligned}
$$

where atan 2 is the two-argument arctangent function. The angle between both symmetric sequences, $\widehat{\varphi}$, will be used to compute the peak current limiter.

\section{Reference Generator}

The reference generator that is used to generate the reference currents is

$$
\begin{aligned}
& i_{\alpha}^{*}=I_{q}^{+} \frac{\widehat{v}_{\beta}^{+}}{\widehat{V}^{+}}+I_{q}^{-} \frac{\widehat{v}_{\beta}^{-}}{\widehat{V}^{-}} \\
& i_{\beta}^{*}=-I_{q}^{+} \frac{\widehat{v}_{\alpha}^{+}}{\widehat{V}^{+}}-I_{q}^{-} \frac{\widehat{v}_{\alpha}^{-}}{\widehat{V}^{-}} .
\end{aligned}
$$

The only difference between (15) and (16) and the conventional reference generator in (4) and (5), is the use of a virtual voltage $\widehat{v}$ instead of the voltage at the terminals of the converter $v$. In the conventional case, the sequence voltages 
$v_{\alpha}^{+}, v_{\beta}^{+}, v_{\alpha}^{-}$and $v_{\beta}^{-}$, and their amplitudes $V^{+}$and $V^{-}$are computed based on a sequence extractor. For the modified version, these values are also obtained with a sequence extractor, but they must be previously estimated with (8) and (9).

\section{Reactive Current Controller}

The next step in the control proposal is the calculation of the amplitude of the reactive currents $I_{q}^{+}$and $I_{q}^{-}$in (15) and (16). As previously mentioned, these magnitudes depend on the control objectives. For the proposed objectives in (1) and (2), the current references are derived from the voltage support analysis as

$$
\begin{aligned}
& I_{q}^{+}=\frac{V_{\mathrm{ref}}^{+}-\widehat{V}^{+}}{\omega \widehat{L}} \\
& I_{q}^{-}=-\frac{V_{\mathrm{ref}}^{-}-\widehat{V}^{-}}{\omega \widehat{L}}
\end{aligned}
$$

where it is shown that the positive sequence current $I_{q}^{+}$ depends on the target voltage $V_{\text {ref }}^{+}=1$ p.u., the virtual voltage $\widehat{V}^{+}$, the grid frequency $\omega$, and the virtual inductor $\widehat{L}$. Similarly, the negative sequence reactive current $I_{q}^{-}$depends on the objective voltage $V_{\text {ref }}^{-}=0$ p.u. and the virtual computation $\widehat{V}^{-}$, the grid frequency $\omega$, and the virtual reactor $\widehat{L}$. Note that (17) and (18) form a proportional controller of the symmetric voltage errors. The gain of these controllers is fixed and depends on the grid frequency and virtual inductance. A discussion on the selection of the virtual inductance $\widehat{L}$, and the closed-loop dynamics of the whole system are presented in Section IV.

The calculation of the reactive currents in (17) and (18), along with the virtual voltage artifact constitute the main contribution of the work since it is now possible to fully cancel the negative sequence at the terminals of the STATCOM by using a simple control based on the stationary reference frame.

\section{E. Peak Current Limiter}

Once the reference current generator, the virtual voltage estimator and the amplitude of the reference currents have been derived, the last remaining step is to provide the STATCOM with a peak current limiter. This limiter must ensure that the phase currents $I_{a}, I_{b}$ and $I_{c}$ are below the rated current $I_{\max }$ of the converter. Also, as previously mentioned, the peak current limiter has a priority-based implementation which is of interest for the operation of the STATCOM whenever there is no enough current capacity to achieve the desired objectives as for example severe voltage perturbations or stiff grids which is the main limitation for voltage regulation when using STATCOMs.

Similarly to [28], [34], the amplitude of each phase current can be expressed as

$$
\begin{aligned}
I_{a} & =\sqrt{\left(I_{q}^{+}\right)^{2}+\left(I_{q}^{-}\right)^{2}+2 I_{q}^{+} I_{q}^{-} \cos \left(\varphi_{I}\right)} \\
I_{b} & =\sqrt{\left(I_{q}^{+}\right)^{2}+\left(I_{q}^{-}\right)^{2}+2 I_{q}^{+} I_{q}^{-} \cos \left(\varphi_{I}+2 \pi / 3\right)} \\
I_{c} & =\sqrt{\left(I_{q}^{+}\right)^{2}+\left(I_{q}^{-}\right)^{2}+2 I_{q}^{+} I_{q}^{-} \cos \left(\varphi_{I}-2 \pi / 3\right)}
\end{aligned}
$$

where $\varphi_{I}=\pi-\widehat{\varphi}$ is the angle between the positive and the negative sequence currents.

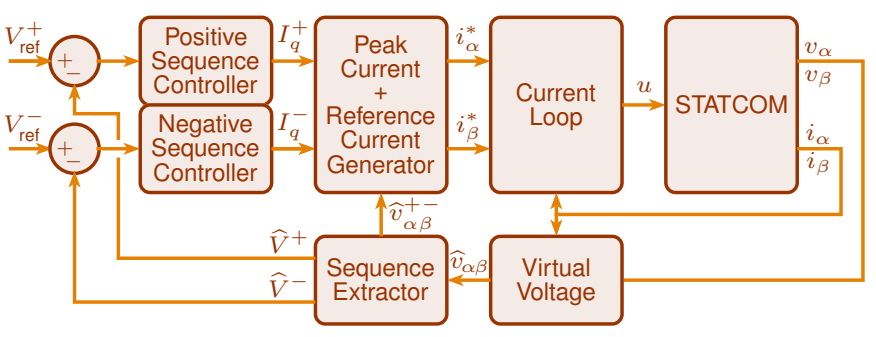

Fig. 3: Diagram for the proposed control scheme with the outer voltage loop, the inner current loop and the plant.

To develop the limiter, assume that the voltage at the terminals of the STATCOM is quite low, and the positive sequence reactive current $I_{q}^{+}$that should be injected is very large. Under such condition, the priority-based behavior of the device should inject only positive sequence current and set the negative sequence reactive current as $I_{q}^{-}=0 \mathrm{~A}$. Solving (19)-(21) for the positive reactive current, and taking into consideration that the injected current should be limited to $I_{\max }$, then the first part of the current limiter is obtained

$$
I_{q}^{+} \leq I_{\max } .
$$

Now assume that the voltage perturbation is less severe, and the first objective in (1) is accomplished. Then, the STATCOM can inject negative sequence current $I_{q}^{-}$to achieve the second objective in (2). Under these conditions, the peak current limiter becomes more complex since the combination of positive and negative sequence currents inherently induces a different amplitude in the injected currents $I_{a} \neq I_{b} \neq I_{c}$ [28]. This characteristic results in the need for computing the maximum negative sequence current $I_{q}^{-}$in the three phases, and then select the minimum value of the three computations. By doing this, the STATCOM will operate safely and will exploit its capabilities to its maximum for any given grid perturbation.

Solving (19)-(21) for the negative sequence reactive current, the following relations are obtained

$$
\begin{aligned}
I_{q_{a} \max }^{-} & =\sqrt{I_{\max }^{2}-\left(I_{q}^{+}\right)^{2} \sin ^{2}(\widehat{\varphi})}-I_{q}^{+} \cos (\pi-\widehat{\varphi}) \\
I_{q_{b} \max }^{-} & =\sqrt{I_{\max }^{2}-\left(I_{q}^{+}\right)^{2} \sin ^{2}(\widehat{\varphi}+2 \pi / 3)}-I_{q}^{+} \cos (\pi-\widehat{\varphi}+2 \pi / 3) \\
I_{q_{c} \max }^{-} & =\sqrt{I_{\max }^{2}-\left(I_{q}^{+}\right)^{2} \sin ^{2}(\widehat{\varphi}-2 \pi / 3)}-I_{q}^{+} \cos (\pi-\widehat{\varphi}-2 \pi / 3) \\
I_{q \max }^{-} & =\min \left\{I_{q_{a} \max }^{-}, I_{q_{b} \max }^{-}, I_{q_{c} \max }^{-}\right\}
\end{aligned}
$$

where $I_{q_{a} \max }^{-}, I_{q_{b} \max }^{-}$and $I_{q_{c} \max }^{-}$are the maximum negative sequence reactive currents available in each phase to avoid surpassing $I_{\max }$, and $I_{q \max }^{-}$takes the minimum of the three computations.

\section{F. Implementation}

In order to further detail the implementation, the control block diagram of the proposed controller is presented in Fig. 3. From this plot, it should be highlighted the block related to the virtual voltage estimator, that captures the measured 


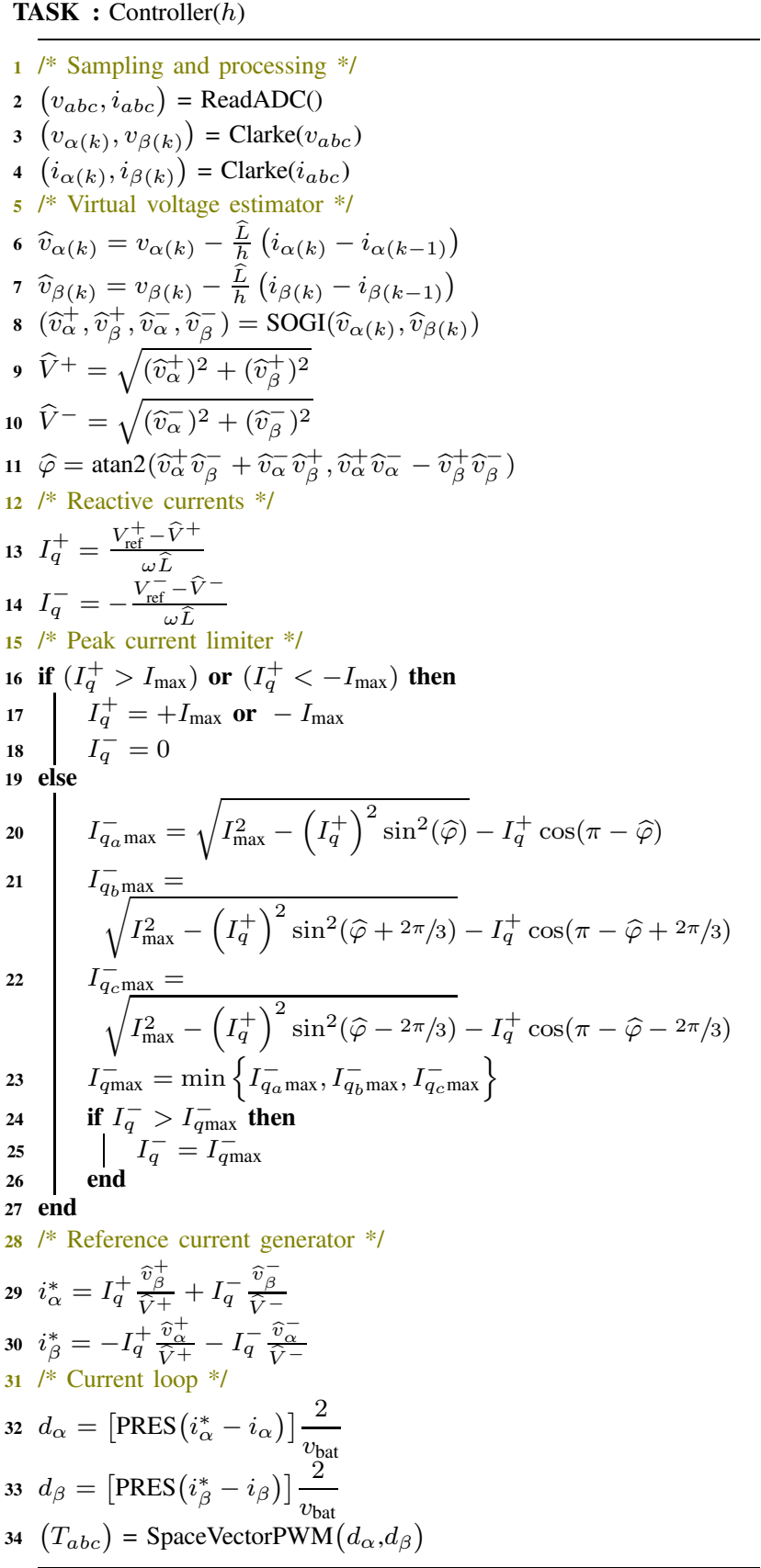

Fig. 4: Pseudo-code of the controller task.

voltages and currents to build the virtual values from (8) and (9). The virtual values will be used in the positive and negative sequence controllers after being processed by the sequence extractor. Two symmetric sequence controllers are used. As previously mentioned, each one of these controllers consists of a proportional term of the voltage error, see (17) and (18). The peak current (22)-(26) and the reference generator (15)-(16) are the last part of the voltage loop. The outputs go directly to the inner current loop of the STATCOM. The current loop joins a proportional+resonant (PRES) controller and a space vector modulator to obtain the control signals to drive the switches of the converter.
Finally, the pseudo-code of the proposal is presented in Fig. 4. The controller algorithm is divided into several parts: sampling and processing, virtual voltage estimator, reactive current calculation, peak current limiter, reference generator and current loop. This pseudo-code presents a complete implementation of the proposal, showing detailed characteristics of the computations required for the whole controller. Note for example that in line number 16 of Fig. 4, the reactive current $I_{q}^{+}$could be negative during voltage swells and need to be checked to ensure the objective presented in (3). Also, it is interesting to note that in lines 24 to 26 , the negative sequence current is limited to avoid over-current tripping, and to implement the priority assignment previously described, which prioritizes the positive sequence regulation in front of the negative sequence voltage support.

\section{Closed-Loop Analysis}

After presenting the main theoretical findings of the paper and the implementation details, one point still remains to be analyzed. This section covers the closed-loop dynamics of the proposed control scheme and concludes with the selection of the virtual inductance $\widehat{L}$ in a real application.

The closed-loop dynamics has been obtained based on the following assumptions: i) other dynamics of the STATCOM are decoupled from the presented closed-loop analysis, and ii) the plant dynamics regarding the voltage support are neglected. The first assumption states that the STATCOM can provide the required reactive power without being affected by the dc-link voltage controller whose dynamics should be adjusted to allow sudden reactive current variations. The second assumption simplifies the analysis by considering that the changes in the reactive currents injected have a fast dynamics that cause the voltage variations almost instantaneously. Therefore, the voltage support equations can be written as

$$
\begin{aligned}
& V^{+}=V_{g}^{+}+\omega L I_{q}^{+} \\
& V^{-}=V_{g}^{-}-\omega L I_{q}^{-}
\end{aligned}
$$

where it can be proved that the injected currents support the voltage (an increment in the positive sequence, and a reduction in the negative one) proportionally to the injected current, the grid frequency and the grid impedance. This is a common assumption in voltage support control analysis.

Based on the control scheme presented in Fig. 3, the transfer functions needed to analyze the closed-loop system are presented in Fig. 5. The positive sequence channel is presented in Fig. 5(a), and the negative sequence blocks are collected in Fig. 5(b). From this figure, the main transfer functions in the Laplace domain are detailed as follows.

The controller transfer function consists on a proportional gain according to (17) and (18), therefore the positive and the negative transfer functions are

$$
G_{\text {control }}^{+}(s)=\frac{I_{q}^{+}(s)}{e^{+}(s)}=\frac{1}{\omega \widehat{L}} \quad, \quad G_{\text {control }}^{-}(s)=\frac{I_{q}^{-}(s)}{e^{-}(s)}=-\frac{1}{\omega \widehat{L}}
$$

The dynamic behavior of the SOGI is complex. However its linearized formulation [35] allows to simplify the dynamics 


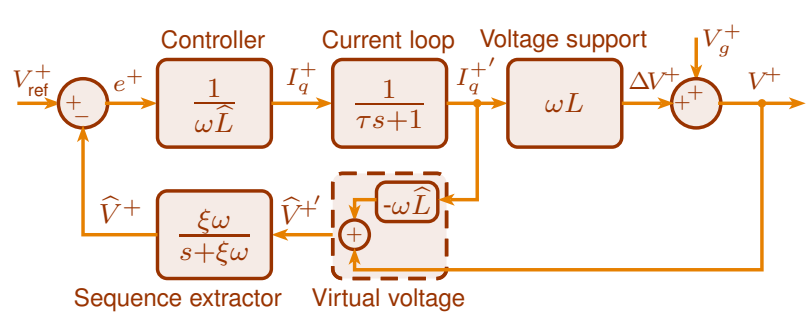

(a)

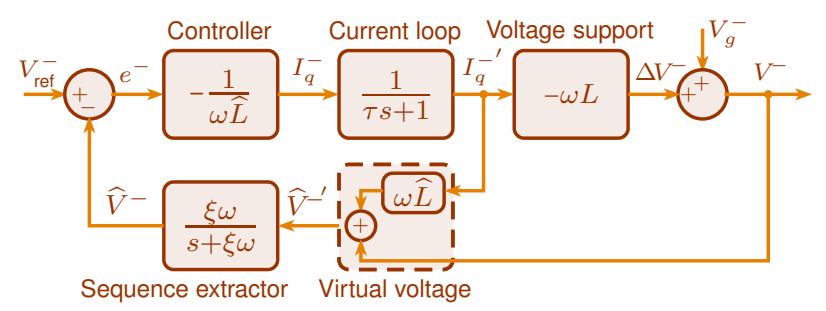

(b)

Fig. 5: Closed-loop transfer functions for the proposed control. (a) Positive sequence channel, (b) negative sequence channel.

both for the positive and negative sequences as a low-pass filter with cutoff frequency equal to $\xi \omega$ as

$$
G_{\text {sogi }}(s)=\frac{\widehat{V}^{+}(s)}{\widehat{V}^{+^{\prime}}(s)}=\frac{\widehat{V}^{-}(s)}{\widehat{V}^{-{ }^{\prime}}(s)}=\frac{\xi \omega}{s+\xi \omega}
$$

where $\xi$ is the selectivity of the filter [33].

A simplified characterization of the the current loop is obtained by considering it as a first order system. This model considers that the current reference from the outer voltage loop takes some time to effectively being injected by the inner current loop into the grid. The positive and negative transfer functions are symmetric and can be represented as

$$
G_{\text {current }}(s)=\frac{I_{q}^{+^{\prime}}(s)}{I_{q}^{+}(s)}=\frac{I_{q}^{-^{\prime}}(s)}{I_{q}^{-}(s)}=\frac{1}{\tau s+1} .
$$

The last part corresponds to the voltage support provided by the STATCOM at the PCC with respect to the grid. The magnitude of this voltage variation has been presented in (27) and (28) where $V_{g}^{ \pm}$are the grid voltages considered as perturbations within the proposed analysis. Based on these relations, the plant is considered as a pure gain

$G_{\text {plant }}^{+}(s)=\frac{\Delta V^{+}(s)}{I_{q}^{+}(s)}=\omega L \quad, \quad G_{\text {plant }}^{-}(s)=\frac{\Delta V^{-}(s)}{I_{q}^{-}(s)}=-\omega L$

Finally, the closed-loop behavior of the virtual estimator is similar to any state estimator whose inputs are the measured plant outputs and the applied control signal, while the output is the estimation value. The following relations are performed inside these blocks

$$
\begin{aligned}
& \widehat{V}^{+^{\prime}}(s)=V^{+}(s)-\omega \widehat{L} I_{q}^{+}(s) \\
& \widehat{V}^{-^{\prime}}(s)=V^{-}(s)+\omega \widehat{L} I_{q}^{-}(s)
\end{aligned}
$$

Joining all these parts together, it is possible to obtain the output dynamics as

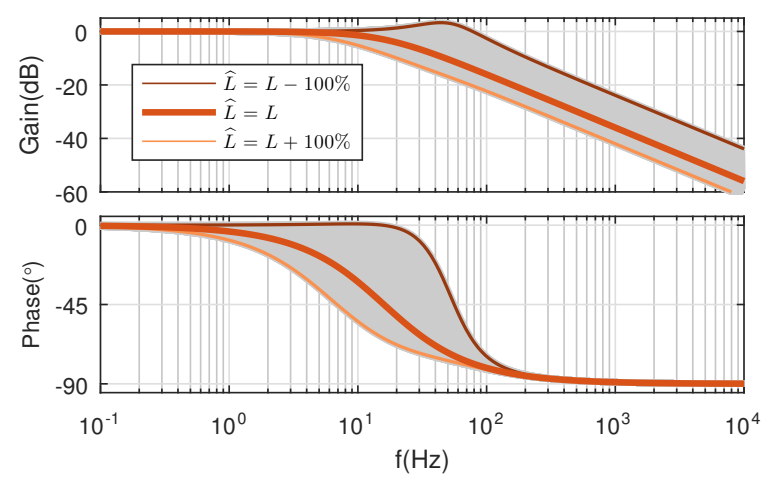

Fig. 6: Bode plot for the closed-loop transfer function $G_{\text {closed-loop }}$

$$
\begin{aligned}
& V^{+}(s)=G_{\text {closed-loop }} V_{\text {ref }}^{+}(s)+G_{\text {perturbation }} V_{g}^{+}(s) \\
& V^{-}(s)=G_{\text {closed-loop }} V_{\text {ref }}^{-}(s)+G_{\text {perturbation }} V_{g}^{-}(s)
\end{aligned}
$$

where

$$
\begin{aligned}
G_{\text {closed-loop }} & =\frac{L s+L \xi \omega}{\widehat{L} \tau s^{2}+(1+\tau \xi \omega) \widehat{L} s+L \xi \omega} \\
G_{\text {perturbation }} & =\frac{\widehat{L} \tau s^{2}+(1+\tau \xi \omega) \widehat{L} s}{\widehat{L} \tau s^{2}+(1+\tau \xi \omega) \widehat{L} s+L \xi \omega}
\end{aligned}
$$

The transfer function in (37) shows the dynamics of the proposed control scheme based on the plant parameters $\omega$ and $L$, the inner current loop characteristics $\tau$, the sequence extractor selectivity $\xi$ and the control parameter $\widehat{L}$. This transfer function has been plotted in Fig. 6 to further detail the main closed-loop characteristics. The plot shows the closedloop bode diagram for different values of the control parameter $\widehat{L}$.

It is clearly shown, both from the analysis of the transfer function in (37), and in the magnitude response of Fig. 6, that

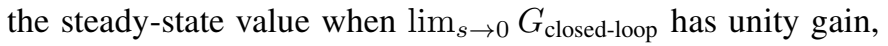
as expected. Therefore, the steady-state does not depend on the virtual inductance which clearly demonstrates the enhanced properties of the proposed closed-loop control. From the closed-loop transfer function, it is interesting to note that the dominant pole, assuming that the inner current loop is fast enough, is located at $s=-\frac{L \xi \omega}{\widehat{L}}$ which makes the system stable provided that $\widehat{L}>0$. As shown in Fig. 6, this dominant pole becomes a complex conjugate for low values of $\widehat{L}$. From (37), this behaviour is reached whenever $\widehat{L}<\frac{4 \xi \omega \tau L}{(1+\xi \omega \tau)^{2}}$.

\section{A. Selection of the control parameter $\widehat{L}$}

In the following, several considerations are presented for the selection of the virtual inductance, including real impedance knowledge, closed-loop dynamics, stiffness/weakness of the grid, and rated voltage and current.

As a first approach, selecting $\widehat{L}=L$ seems to be the best option. However, the real grid impedance can not be perfectly known due to daily variations or even the fault location. However, an approximate knowledge, although inaccurate, is always available when commissioning the STATCOM, and this 
value will serve as the starting point for the design of the control parameter.

Due to the uncertainty in the grid impedance, it is required to analyze the effects on the closed-loop dynamics. Assuming that $\widehat{L}$ has been selected based on a bad estimation of the real grid impedance, the transient dynamics is affected as follows. In case that the virtual value is larger than the real impedance, the dynamics is slow-down. Similarly, for smaller virtual than real inductance, the dynamics is speed-up. This effect can be appreciated in Fig. 6 for $\widehat{L}=L+100 \%$ and $\widehat{L}=L-100 \%$ respectively where the dominant pole move towards lower frequencies as $\widehat{L}$ increases. Based on this information, it can be concluded that $\frac{\widehat{L}}{L}>1$ is of interest to reduce faster dynamics and avoid overshoots which are not the preferred option when supporting the voltage. However, this value should not be very large in order to provide an admissible settling time $t_{s} \approx 4 \frac{\widehat{L}}{L \xi \omega}$ during the perturbation.

Regarding the stiffness/weakness of the grid, the selection of the virtual inductance has no noticeable impact. Apart from requiring more or less current to achieve the voltage objectives, the controller performs as expected in any of these conditions. This property should be highlighted since it is possible to adapt the proposed controller to different network requirements.

Similarly, the rated voltage barely affects the proposed control. However, the rated capacity of the STATCOM must be taken into account. The reason for this is that typically, the higher the rated current, the lower the speed response of the current loop which is considered within the parameter $\tau$ in (31), and it is modelled in the transfer function (37). Therefore, to fastly react to grid perturbations as voltage dips, the current loop and the desired closed-loop dynamics should be fast enough. Otherwise, the STATCOM would not be capable to counteract these perturbations.

The aforementioned analysis of the static and dynamic behavior of the proposed control scheme yields to a simplified design guideline for the control parameter $\widehat{L}$. The control design starts by identifying an approximate value of the real grid impedance $L$. Since this value can not be perfectly known, a trade-off between settling time and desired dynamics is required taking into account the uncertainty introduced by the plant parameter. By choosing $\frac{\widehat{L}}{L}>1$, the system slows down and the response time increases. By choosing $\frac{\widehat{L}}{L} \approx 1$, the response time is reduced and the system is fast enough to counteract voltage dips and any other perturbation. The selection of $\frac{\widehat{L}}{L}<1$ is not recommended in any case although it does not compromise the stability. This guideline is simple and easy to implement, which is an important characteristic of the proposed control scheme that makes the proposal a good candidate to improve the power quality of the grid.

\section{EXPERIMENTAL RESULTS}

The control proposal has been tested in a laboratory prototype. The test-bench mimics the scheme shown in Fig. 1. It is made up of an Amrel DC power source to emulate the dc-link, a Guasch three-phase voltage source converter limited to $I_{\max }=10 \mathrm{~A}$, and a Pacific Power AC grid emulator. The grid impedance has been selected so as to emulate a weak grid
TABLE II: System and Control Parameters

\begin{tabular}{lccc}
\hline \hline & Symbol & Nominal value & Per unit \\
\hline base power & $S_{\text {base }}$ & $3.5 \mathrm{kVA}$ & 1 \\
rated current & $I_{\max }$ & $10 \mathrm{~A}$ (peak) & 1 \\
grid voltage & $v_{g}$ & $155 \mathrm{~V}(1-\mathrm{n}$, peak) & 1 \\
grid frequency & $\omega$ & $2 \pi 60 \mathrm{rad} / \mathrm{s}$ & 1 \\
dc-link voltage & $v_{\text {bat }}$ & $350 \mathrm{~V}$ & - \\
grid inductance & $L$ & $5 \mathrm{mH}$ & 0.12 \\
converter inductance & $L_{i}$ & $5 \mathrm{mH}$ & 0.12 \\
nominal load & $R$ & $22 \Omega$ & 1.4 \\
switching frequency & $f_{s}$ & $10 \mathrm{kHz}$ & - \\
sampling period & $h$ & $100 \mu \mathrm{s}$ & - \\
pos. seq. voltage ref. & $V_{\text {ref }}^{+}$ & $155 \mathrm{~V}$ & 1 \\
neg. seq. voltage ref. & $V_{\text {ref }}^{-}$ & $0 \mathrm{~V}$ & 0 \\
virtual inductance & $\hat{L}$ & $7.5 \mathrm{mH}$ & 0.18 \\
SOGI selectivity & $\xi$ & 0.7 & - \\
PRES prop. gain & $k_{p}$ & 40 & - \\
PRES resonant gain & $k_{r}$ & 200 & - \\
\hline
\end{tabular}

connection ( $L=0.12$ p.u.). This scenario has been selected as the worst case for a STATCOM control, since these devices can be attached to remote locations where the grid is weak and the voltage regulation is a must. Even though, it should be noted that the performance of the proposed controller does not depend of the stiffness or weakness of the grid, since the peak current limiter and the priority assigned to each one of the voltage targets, makes the proposal independent of the grid impedance. Regarding the virtual inductance, and according to the analysis previously presented, it has been selected as $\widehat{L}=1.5 \mathrm{~L}$. This value has been selected according to the previous design criterion and to demonstrate that even for a mismatch of $50 \%$ in the knowledge of the grid impedance value, the proposed controller performs well. The main system and control parameters are collected in Table II.

The controller is implemented on a Texas Instruments F28M36 digital signal processor, according to the pseudo-code in Fig. 4. The sequence extractor and the resonant controllers for the current loop are based on SOGIs. The current loop also includes a space vector modulator to compute the switching times of each converter branch.

In order to demonstrate the performance of the controller in any type of grid perturbation, a complex test has been developed. The experiment covers the most diverse conditions for the device under test, including some imbalance, a voltage dip, the dip recovery and the connection of an unbalanced load. Phase voltages and currents are collected with a Yokogawa DL850 oscilloscope and exported to Matlab from representation, while the rest of the signals are directly obtained from the microcontroller. The test has been divided into 5 regions, each one lasting 0.1 seconds. The voltage measured at the PCC when the STATCOM is unplugged can be seen in Fig. 7.

In the top plot of Fig. 7, it is shown a small voltage imbalance programmed from $t=0.1 \mathrm{~s}$ to $t=0.2 \mathrm{~s}$. In this region, the controller should cancel completely the negative sequence voltage.

The second part of the test, between $t=0.2 \mathrm{~s}$ and $t=0.3$ $\mathrm{s}$, corresponds to a voltage dip. In this case, positive sequence current injection is required to support as much as possible 


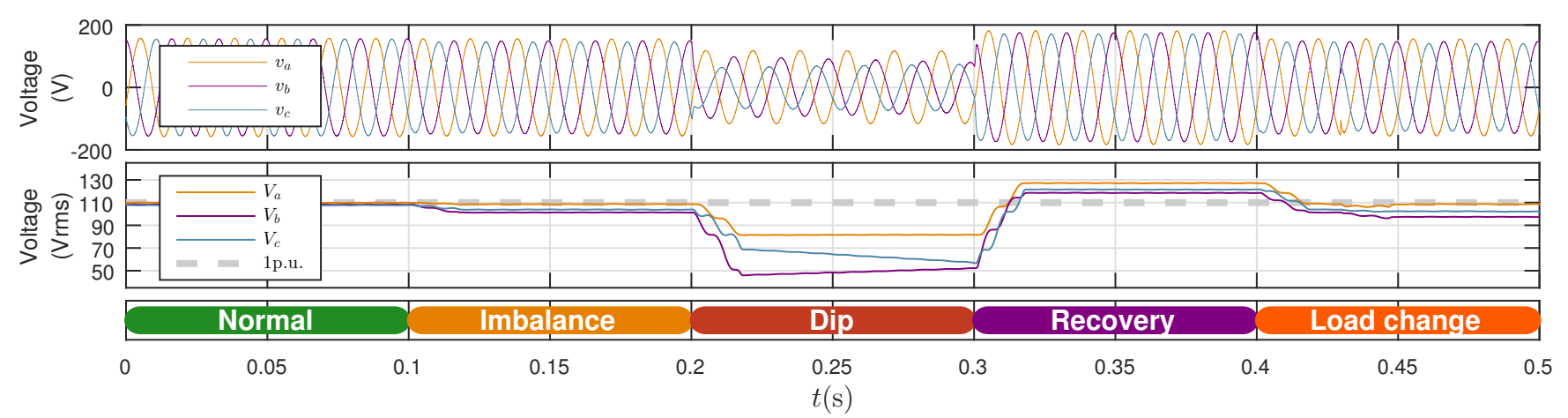

Fig. 7: Experimental measurements for the test scenario with the STATCOM unplugged. From top to bottom: phase voltages, rms voltages and test condition.

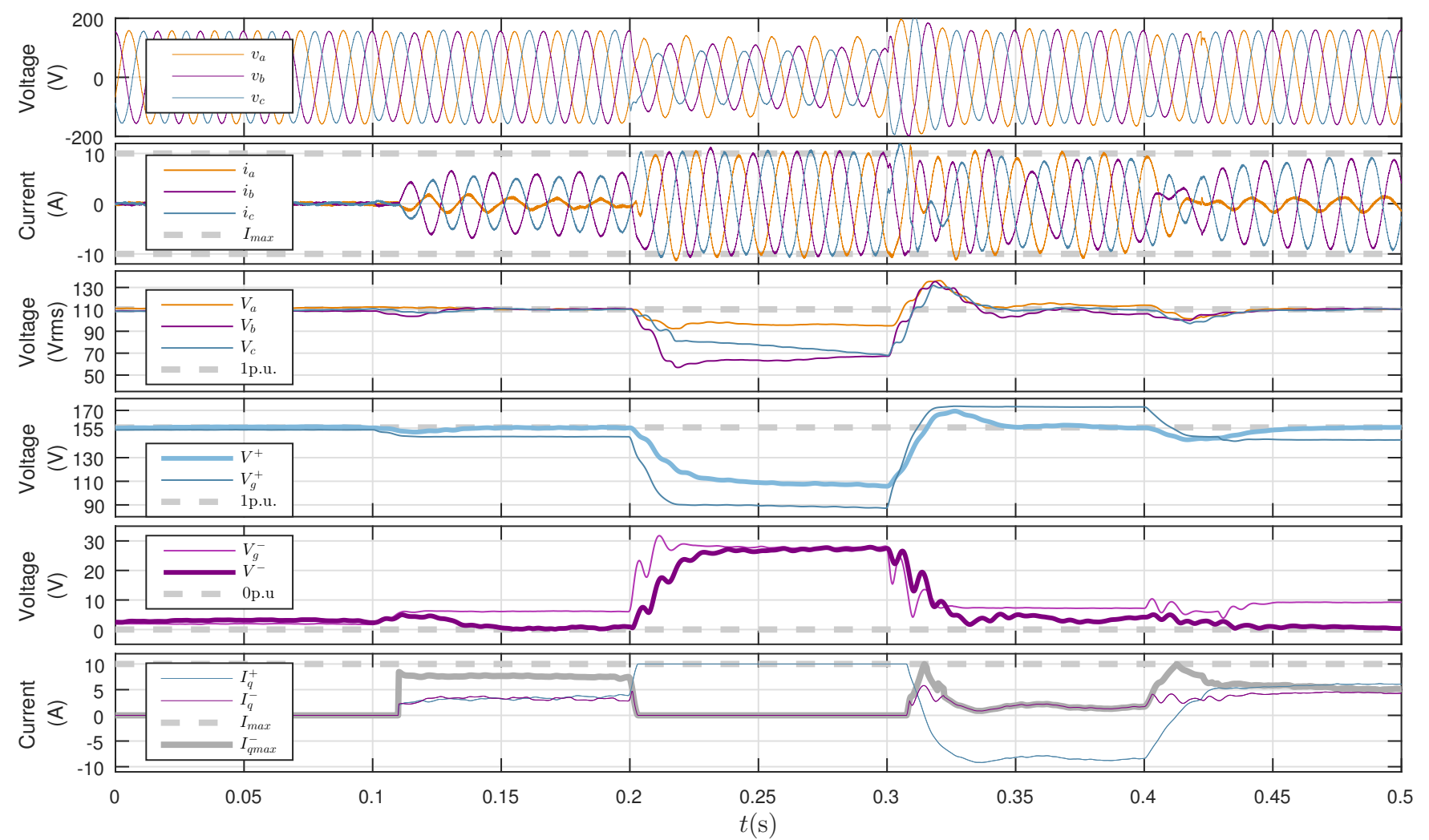

Fig. 8: Experimental results for the proposed control. From top to bottom: phase voltages, phase currents, rms voltages, positive sequence voltage, negative sequence voltage and reactive currents.

the positive sequence voltage, while supporting the negative sequence voltage is a non-prioritary objective. In this part of the test, the peak current limiter should activate and inject the maximum rated current of the STATCOM for voltage support.

The third zone occurs between $t=0.3 \mathrm{~s}$ and $t=0.4 \mathrm{~s}$. During this interval, the voltage dip has been recovered and a typical over-voltage phenomenon is observed. The controller in such a situation should reduce the high positive sequence voltage, and the rest of the current will be used to reduce the unbalanced voltage, limiting the injected current in case that the STATCOM has not enough capacity.

The last part of the experiment, between $t=0.4 \mathrm{~s}$ and $t=$ $0.5 \mathrm{~s}$, explores the controller capabilities when an unbalanced local load is connected at the PCC. In this case, the voltage imbalance increases slightly and the controller needs to fully cancel this imbalance. The load changes from $R_{a}=R_{b}=$ $R_{c}=22 \Omega$ to $R_{a}=R_{c}=11 \Omega$ and $R_{b}=22 \Omega$.

Fig. 8 shows the experimental results obtained during the experiment when the STATCOM is activated. The plot is divided into six parts showing the most significant magnitudes.

The top plot in Fig. 8 shows the instantaneous phase voltages at the PCC. Comparing the voltages in the previous figure (unplugged STATCOM) and the results in this figure (active STATCOM), it is shown the voltage regulating capabilities. Along the experiment the voltages tend to be closer to the nominal value. During the dip and the recovery $(0.2<t \leq 0.4$ s), the voltages are far from the target objectives. However, a noticeable voltage support is observed due to the weak grid conditions.

The next plot in Fig. 8 presents the currents injected by 
the STATCOM. In the second part of the experiment $(0.1<$ $t \leq 0.2 \mathrm{~s})$, the currents are unbalanced to simultaneously comply with the voltage objectives. After that, during the voltage dip, the peak current limiter is activated, and the maximum rated current of the converter is injected. Since the first positive sequence voltage objective has higher priority, only positive sequence reactive current is required, and the currents are perfectly balanced and limited to $I_{\max }$. Next step $(0.3<t \leq 0.4 \mathrm{~s})$ corresponds to the post-fault recovery, and typically an over-voltage situation can be manifested. Due to the high voltage, there is no enough current to fully cancel the negative sequence voltage, and the peak current limiter is activated again. Note that the current in phase $\mathrm{A}$ is safely limited to $10 \mathrm{~A}$. Finally, at $(0.4<t \leq 0.5 \mathrm{~s})$, the fault has been perfectly cleared and an unbalanced load is connected at the PCC, causing a greater imbalance. In such a situation, the STATCOM is capable to regulate the positive sequence voltage to the nominal value, and to completely cancel the negative sequence voltage.

The third plot presents the rms magnitudes of the phase voltages. It is clearly shown the almost ideal voltage regulation capabilities of the controller, except in the region where the peak current limiter is activated because the converter has no enough capacity to achieve the voltage objectives.

The fourth and fifth plots present the positive and the negative sequence voltages. Also, it has been included the magnitudes when the STATCOM is deactivated in thin lines for comparison. The most important part of this graph is the full cancellation of the negative sequence voltage, which confirms the expected power quality improvement with the proposed control method.

Finally, the reactive currents injected are presented in the last plot. This plot shows the evolution during the different experiment parts, and the limitation when the peak current is activated. Also, in grey lines, the maximum rated current of the converter and the maximum allowable negative sequence currents are plotted for comparison. As an interesting detail, note that the current $I_{q}^{+}$will be negative during the recovery part of the experiment. Also note that when $I_{q}^{-}$is lower than $I_{q^{-} \text {max }}$, the peak current limitation is not activated and the negative sequence voltage can be completely eliminated. Conversely, when $I_{q}^{-}$coincides with $I_{q^{-} \text {max }}$ (during the voltage dip and the recovery intervals), the peak current limitation is activated in order to safely operate the STATCOM.

\section{CONCLUSIONS}

This paper has presented a STATCOM control with three main objectives: positive sequence voltage regulation, full negative sequence cancellation, and peak current limitation with priorities. The main contribution of the presented work is the inclusion of these three control objectives simultaneously in a low complexity, easy to tune implementation.

The solution proposed can help to improve the regulation capabilities of STATCOMs, being the full cancellation of the voltage imbalance an interesting ancillary service for a new generation of STATCOMs. The control is suitable for the operation during voltage dips or swells, and stiff or weak grid connections due to the priority-based peak current limiter. The main drawback of the proposed control scheme is the approximate knowledge of the grid impedance which makes the proposal dependent of this value, although it has been shown that the system presents good transient dynamics and steady-state behaviour even during not perfectly known grid impedance values.

Future work will focus on the integration of the proposed controller into complex systems, and the interaction with distributed generation sources implementing low voltage ride through requirements. Also, the coordination with planning and dispatching schedulers should be taken into account for the proper operation and optimization of the whole system.

\section{REFERENCES}

[1] "New ERA for electricity in Europe. Distributed Generation: Key issuses, challenges and proposed solutions," European Commission, Energy Production and Distribution Systems, Rep., 2003.

[2] "The potential benefits of distributed generation and rate-related issues that may impede their expansion," U.S. Department of Energy, Rep., Feb. 2007.

[3] F. Blaabjerg, Z. Chen, and S. Kjær, "Power electronics as efficient interface in dispersed power generation systems," IEEE Trans. Power Electron., vol. 19, no. 5, pp. 1184-1194, Sep. 2004.

[4] G. Wang, G. Konstantinou, C. D. Townsend, J. Pou, S. Vazquez, G. D. Demetriades, and V. G. Agelidis, "A review of power electronics for grid connection of utility-scale battery energy storage systems," IEEE Trans. Sustain. Energy, vol. 7, no. 4, pp. 1778-1790, Oct. 2016.

[5] A. Yazdani and R. Iravani, Voltage-Sourced Converters in Power Systems. New Jersey, USA: Wiley-IEEE Press, 2010.

[6] B. Kirby, "Ancillary services: Technical and commercial insights," Wartsila North America Inc., Rep., Jun. 2007.

[7] Q. C. Zhong, "Power-electronics-enabled autonomous power systems: Architecture and technical routes," IEEE Trans. Ind. Electron., vol. 64, no. 7, pp. 5907-5918, Jul. 2017.

[8] Y. N. S. H. Hosseini, S. G. Zadeh, B. Mohammadi-Ivatlo, J. C. Vasquez, and J. M. Guerrero, "Distributed power quality improvement in residential microgrids," in 10th Int. Conf. Elect. Electron. Eng., Nov. 2017, pp. 90-94.

[9] J. H. Dymond and N. Stranges, "Operation on unbalanced voltage: One motor's experience and more," IEEE Trans. Ind. Appl, vol. 43, no. 3, pp. 829-837, May 2007.

[10] J. Faiz, H. Ebrahimpour, and P. Pillay, "Influence of unbalanced voltage on the steady-state performance of a three-phase squirrel-cage induction motor," IEEE Trans. Energy Convers., vol. 19, no. 4, pp. 657-662, Dec. 2004.

[11] A. von Jouanne and B. Banerjee, "Assessment of voltage unbalance," IEEE Trans. Power Del., vol. 16, no. 4, pp. 782-790, Oct. 2001.

[12] K. H. Chua, Y. S. Lim, P. Taylor, S. Morris, and J. Wong, "Energy storage system for mitigating voltage unbalance on low-voltage networks with photovoltaic systems," IEEE Trans. Power Del., vol. 27, no. 4, pp. 1783-1790, Oct. 2012.

[13] T. Lee, S. Hu, and Y. Chan, "D-STATCOM with positive-sequence admittance and negative-sequence conductance to mitigate voltage fluctuations in high-level penetration of distributed generation systems," IEEE Trans. Ind. Electron., vol. 60, no. 4, pp. 1417-1428, Apr. 2013.

[14] K. Li, J. Liu, Z. Wang, and B. Wei, "Strategies and operating point optimization of STATCOM control for voltage unbalance mitigation in three-phase three-wire systems,"' IEEE Trans. Power Del., vol. 22, no. 1, pp. 413-422, Jan. 2007.

[15] M. Savaghebi, A. Jalilian, J. C. Vasquez, and J. M. Guerrero, "Autonomous voltage unbalance compensation in an islanded droopcontrolled microgrid," IEEE Trans. Ind. Electron., vol. 60, no. 4, pp. 1390-1402, Apr. 2013.

[16] A. Moawwad, V. Khadkikar, and J. L. Kirtley, "Interline photovoltaic (I-PV) power plants for voltage unbalance compensation," in 38th Ann. Conf. Ind. Electron. Society, Oct. 2012, pp. 5330-5334.

[17] Y. Wang, L. Xu, and B. W. Williams, "Compensation of network voltage unbalance using doubly fed induction generator-based wind farms," IET Renew. Power Gen., vol. 3, no. 1, pp. 12-22, Mar. 2009. 
[18] A. Rodríguez, E. J. Bueno, A. Mayor, F. J. Rodríguez, and A. GarcíaCerrada, "Voltage support provided by STATCOM in unbalanced power systems," Energies, vol. 7, no. 2, pp. 1003-1026, 2014.

[19] J. Kang, D. Han, Y. Suh, B. Jung, J. Kim, J. Park, and Y. Choi, "Negative sequence current injection control algorithm compensating for unbalanced PCC voltage in medium voltage PMSG wind turbines," in Int. Power Electron. Conf., May 2014, pp. 1185-1192.

[20] H. Nian, T. Wang, and Z. Q. Zhu, "Voltage imbalance compensation for doubly fed induction generator using direct resonant feedback regulator," IEEE Trans. Energy Convers., vol. 31, no. 2, pp. 614-626, Jun. 2016.

[21] A. El-Naggar and I. Erlich, "Control approach of three-phase grid connected PV inverters for voltage unbalance mitigation in low-voltage distribution grids," IET Renew. Power Gen., vol. 10, no. 10, pp. 15771586, 2016.

[22] J. M. Rey, M. Castilla, J. Miret, M. Velasco, P. Mart

and E. Mojica-Nava, "Negative-sequence voltage elimination for distributed generators in grid-feeding operation mode," IET Power Electron., vol. 13, no. 9, pp. 1764-1774, 2020.

[23] A. Borrell, M. Velasco, J. Miret, A. Camacho, P. Marti, and M. Castilla, "Collaborative voltage unbalance elimination in grid-connected ac microgrids with grid-feeding inverters," IEEE Trans. Power Electron., pp. $1-1,2020$.

[24] P. Rodriguez, G. Medeiros, A. Luna, M. C. Cavalcanti, and R. Teodorescu, "Safe current injection strategies for a STATCOM under asymmetrical grid faults," in IEEE Energy Convers. Congr. and Expo., Sep. 2010, pp. 3929-3935.

[25] J. Miret, A. Camacho, M. Castilla, J. L. G. de Vicuña, and J. de la Hoz "Reactive current injection protocol for low-power rating distribute generation sources under voltage sags," IET Power Electron., vol. 8 , no. 6, pp. 879-886, 2015.

[26] M. Mirhosseini, J. Pou, and V. G. Agelidis, "Individual phase current control with the capability to avoid overvoltage in grid-connected photovoltaic power plants under unbalanced voltage sags," IEEE Trans. Power Electron., vol. 30, no. 10, pp. 5346-5351, Oct. 2015.

[27] J. L. Sosa, M. Castilla, J. Miret, J. Matas, and Y. A. Al-Turki, "Control strategy to maximize the power capability of PV three-phase inverters during voltage sags," IEEE Trans. Power Electron., vol. 31, no. 4, pp. 3314-3323, Apr. 2016

[28] A. Camacho, M. Castilla, J. Miret, A. Borrell, and L. G. de Vicuña "Active and reactive power strategies with peak current limitation for distributed generation inverters during unbalanced grid faults," IEEE Trans. Ind. Electron., vol. 62, no. 3, pp. 1515-1525, Mar. 2015.

[29] A. Milicua, G. Abad, and M. A. R. Vidal, "Online reference limitation method of shunt-connected converters to the grid to avoid exceeding voltage and current limits under unbalanced operation; part i: Theory," IEEE Trans. Energy Convers., vol. 30, no. 3, pp. 852-863, Sep. 2015.

[30] W. C. Duesterhoeft, M. W. Schulz, and E. Clarke, "Determination of instantaneous currents and voltages by means of alpha, beta, and zero components," Trans. Am. Inst. Elec. Eng., vol. 70, no. 2, pp. 1248-1255, 1951.

[31] C. L. Fortescue, "Method of symmetrical co-ordinates applied to the solution of polyphase networks," Trans. Am. Inst. Elec. Eng., vol. XXXVII, no. 2, pp. 1027-1140, Jul. 1918

[32] Z. Xin, R. Zhao, P. Mattavelli, P. C. Loh, and F. Blaabjerg, "Reinvestigation of generalized integrator based filters from a first-ordersystem perspective," IEEE Access, vol. PP, no. 99, pp. 1-1, 2016.

[33] F. J. Rodriguez, E. Bueno, M. Aredes, L. G. B. Rolim, F. A. S. Neves, and M. C. Cavalcanti, "Discrete-time implementation of second order generalized integrators for grid converters," in 34th Anпu. Conf. of IEEE Ind. Electron., Nov. 2008, pp. 176-181.

[34] T. Wijnhoven, G. Deconinck, T. Neumann, and I. Erlich, "Control aspects of the dynamic negative sequence current injection of type 4 wind turbines," in IEEE PES Gen. Meet. Conf. Expo., Jul. 2014, pp. $1-5$.

[35] M. Castilla, J. Miret, A. Camacho, L. García de Vicuña, and J. Matas, "Modeling and design of voltage support control schemes for threephase inverters operating under unbalanced grid conditions," IEEE Trans. Power Electron., vol. 29, no. 11, pp. 6139-6150, Nov. 2014.

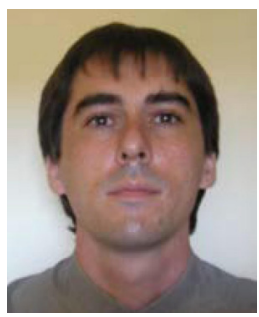

Antonio Camacho received the B.S. degree in chemical engineering, the M.S. degree in automation and industrial electronics, and the Ph.D. degree in electronic engineering from the Technical University of Catalonia, Barcelona, Spain in 2000, 2009 and 2015 respectively. His research interests include networked and embedded control systems, industrial informatics, and power electronics.

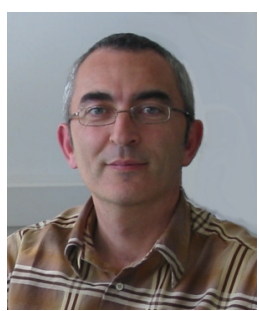

Miguel Castilla received the B.S., M.S., and Ph.D. degrees in telecommunication engineering from the Technical University of Catalonia, Barcelona, Spain, in 1988, 1995, and 1998, respectively. Since 2019, he has been a Full Professor with the Department of Electronic Engineering, Technical University of Catalonia, where he teaches courses on control of power electronic converters. His research interests include the areas of power electronics, control, renewable energy systems, and electrical microgrids.

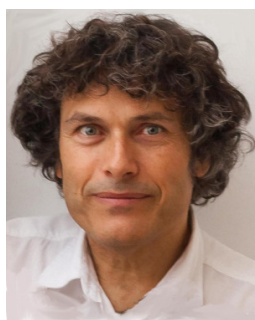

Jaume Miret (M'98) received the B.S. degree in telecommunications, M.S. degree in electronics, and Ph.D. degree in electronics from the Universitat Politecnica de Catalunya, Barcelona, Spain, in 1992 1999, and 2005, respectively. From 1993 to 2011, he was an Assistant Professor in the Department of Electronic Engineering, Universitat Politecnica de Catalunya, Spain. Since 2011 he has been an Associate Professor in the Universitat Politecnica de Catalunya, where he teaches courses on digital design and circuit theory. His research interests include dc-to-ac converters, active power filters, and digital control.

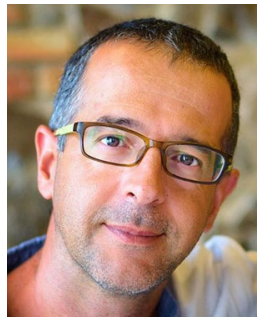

Manel Velasco received the graduate degree in martime engineering and the Ph.D. degree in automatic control from the Technical University of Catalonia, Barcelona, Spain, in 1999 and 2006, respectively. Since 2002, he has been an Assistant Professor with the Department of Automatic Control, Technical University of Catalonia. His research interests include artificial intelligence, real-time control systems, and collaborative control systems, especially on redundant controllers and multiple controllers with self-interacting systems.

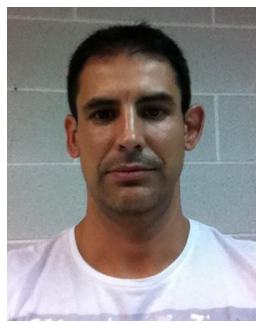

Ramon Guzman received the B.S., the M.S. and the Ph.D. degrees in telecommunications engineering from the Technical University of Catalonia, Barcelona, Spain, in 1999, 2004 and 2016, respectively. He is currently an Associate Professor with the Department of Automatic Control in the Technical University of Catalonia. His research interests include nonlinear and adaptive control for threephase power converters. 